\title{
Lattice NRQCD study of quarkonium at non-zero temperature
}

\section{Seyong Kim*}

Department of Physics, Sejong University, Seoul 143-747, Korea

E-mail: skimesejong.ac.k

\section{Peter Petreczky}

Physics Department, Brookhaven National Laboratory, Upton, NY11973, USA

E-mail: petreczk@quark.phy.bnl.gov

\author{
Alexander Rothkopf \\ Institute for Theoretical Physics, Heidelberg University, Philosophenweg 16, 69120 Heidelberg, \\ Germany \\ E-mail: rothkopfethphys.uni-heidelberg.de
}

To study the in-medium modification of quarkonium properties, charmonium correlators at $140.4(\beta=6.664) \leq T \leq 221(\beta=7.280)(\mathrm{MeV})$ are calculated using the NRQCD formalism on $48^{3} \times 12$ gauge configurations with dynamical $N_{f}=2+1$ flavors of Highly Improved Staggered Quarks (HISQ). To determine the "zero energy shift" for these lattices, we perform a fine zero temperature scan $(\beta=6.664,6.740,6.800,6.880,6.950,7.030,7.150$ and 7.280). We find that the temperature dependence of charmonium correlators is stronger than the temperature dependence of bottomonium correlators in a given channel. This fits into the expected pattern of sequential quarkonium melting.

The 33rd International Symposium on Lattice Field Theory

14 -18 July 2015

Kobe International Conference Center, Kobe, Japan*

${ }^{*}$ Speaker. 


\section{Introduction}

Quarkonium is expected to play an important role as an indicator for quark-gluon plasma (QGP) formation [1]. A quantitative understanding of how quarkonium behaves around the transition temperature based on first principle calculations is a long sought goal of many studies. Recently, bottomonium correlators were studied within a lattice formulation of non-relativistic QCD (NRQCD) in the background of gauge fields simulated in a non-zero temperature environment with dynamical quarks. Spectral functions of various channels have been reconstructed with Bayesian methods and were scrutinized for in-medium modification $[2,3,4,5,6,7,8]$. There are several advantages of this approach: (1) NRQCD is an effective field theory of QCD in which momenta above the soft scale $m v$ including those of the order of the heavy quark rest mass are "integrated out". It represents a systematic series expansion in powers of $v$ (heavy quark velocity in the rest frame of quarkonium) [9]. The difficulty associated with the lattice conditions for a heavy quark that $a$ (lattice spacing) $\ll \frac{1}{M} \ll L a$ (lattice extent) is turned into an advantage as basis of an effective description. (2) The kernel in the spectral representation of NRQCD quarkonium correlators is temperature independent and the problematic constant contribution to the spectral function in relativistic QCD $[10,11,12]$ is absent (note that quarkonium state itself is not in thermal equilibrium). Note also that NRQCD calculations of heavy quark propagators constitute an initial value problem, not a boundary value problem like in the case of a relativistic calculation. This reduces the computational effort significantly.

The studies in Refs. [2, 3, 4, 5, 6] use anisotropic lattices with a fixed lattice spacing (at a somewhat heavy pion mass of $\sim 400 \mathrm{MeV}$ ) and the system temperature is increased by decreasing the number of Euclidean time slices. They employ the Maximum Entropy Method (MEM) [13] for spectral function reconstructions. The work in Ref. [8] uses isotropic lattices with variable lattice spacing (at nearly physical pion and kaon masses). The system temperature is increased by decreasing the lattice spacing. In Ref. [8] both MEM and a new Bayesian Reconstruction (BR) method [14] are used for spectral function reconstruction.

These two studies use different lattice setups and employ different spectral reconstruction methods. A comparison of such different approaches may give us better understanding on the reconstruction uncertainties of spectral functions via Bayesian methods in general. In both [3,6] and [8] a consistent "sequential suppression" pattern of $\Upsilon(1 S), \Upsilon(2 S)$ and $\Upsilon(3 S)$ state has been observed in spectral functions of the $\Upsilon$ channel, which were reconstructed both with MEM and the BR method. Thus lattice NRQCD studies of S-wave bottominum at $T \neq 0$ are on firm grounds. On the other hand for P-wave bottomonium, the spectral functions reconstructed with MEM show a "melting" behavior immediately close to $T_{c}[5,6,8]$ while those reconstructed with the BR method show survival of peaked structures for the ground state up to $1.61 T_{c}$.

Here, we report on preliminary results from our study of in-medium charmonium using the $\mathscr{O}\left(v^{4}\right)$ NRQCD Lagrangian for the calculation of correlator at $T \neq 0$. We use the same $48^{3} \times 12$ gauge configurations generated by HotQCD collaboration $[15,16]$ as in our bottomonium study [8]). The lattice NRQCD parameters for $T \neq 0$ are listed in Table 1 (see [15] for the details on how the lattice gauge fields are generated). At $\beta=6.664,6.800,6.950$ (on $32^{3} \times 32$ latices), $\beta=$ $6.740,6.880,7.030$ (on $48^{3} \times 48$ lattice) and $\beta=7.150,7.280$ (on $48^{3} \times 64$ lattice), accompanying $T=0$ calculation are performed. Since $M_{c} a<1$, we tested the value $n=6$ and 8 for the Lepage 


\begin{tabular}{ccccccc}
\hline \hline$M_{b} a$ & $M_{c} a$ & $\beta$ & $\mathrm{T}(\mathrm{MeV})$ & $\mathrm{T} / T_{c}$ & $a_{\tau}^{-1}(\mathrm{fm})$ & No. of Conf.(analyzed) \\
\hline 2.759 & 0.7566 & 6.664 & 140.40 & 0.911 & 0.1169 & 400 \\
2.667 & 0.7314 & 6.700 & 145.32 & 0.944 & 0.1130 & 400 \\
2.566 & 0.7035 & 6.740 & 150.97 & 0.980 & 0.1087 & 400 \\
2.495 & 0.6841 & 6.770 & 155.33 & 1.008 & 0.1057 & 400 \\
2.424 & 0.6657 & 6.800 & 159.33 & 1.038 & 0.1027 & 400 \\
2.335 & 0.6403 & 6.840 & 165.95 & 1.078 & 0.09893 & 400 \\
2.249 & 0.6167 & 6.880 & 172.30 & 1.119 & 0.09528 & 400 \\
2.187 & 0.5996 & 6.910 & 177.21 & 1.151 & 0.09264 & 400 \\
2.107 & 0.5776 & 6.950 & 183.94 & 1.194 & 0.08925 & 400 \\
2.030 & 0.5566 & 6.990 & 190.89 & 1.240 & 0.08600 & 400 \\
1.956 & 0.5364 & 7.030 & 198.08 & 1.286 & 0.08288 & 400 \\
1.835 & 0.5030 & 7.100 & 211.23 & 1.371 & 0.07772 & 400 \\
1.753 & 0.4806 & 7.150 & 221.08 & 1.436 & 0.07426 & 400 \\
1.559 & 0.4274 & 7.280 & 248.63 & 1.614 & 0.06603 & 400 \\
\hline
\end{tabular}

Table 1: Summary of the lattice parameters of the HotQCD $N_{f}=2+1$ HISQ lattices used in our study. Values of $\beta$ at which $T=0$ correlators are available are emphasized by a light blue backgorund.

parameter and the result from $n=6$ is used for the following analysis.

\section{Preliminary Result}

To determine the "zero energy shift" in NRQCD spectra, we need $T=0$ simulations at each $\beta$. Note that relativistic corrections are expected to be larger in the charmonium system compared to bottomonium, and thus the $\mathrm{T}=0$ masses obtained there might differ significantly from their PDG value. Fig. 1 shows $J / \psi$ correlators at $T=0$ (right) and compares them to $\Upsilon$ correlators (left). Fig. 2 shows $\chi_{c 1}$ correlators (right) and compares them to $\chi_{b 1}$ correlators (left). Both S- and Pwave charmonium NRQCD correlators at $T=0$ follows an exponentially falling behavior and both exponential fits to the correlators or the peak position from spectral functions reconstructed with Bayesian methods give reasonable $\chi_{c 1}-J / \psi$ splitting $\left(\sim 450 \mathrm{MeV}\right.$, compared to $\left(\chi_{c 1}-J / \psi\right)_{\exp } \simeq$ $413 \mathrm{MeV})$.

Fig. 3 shows the ratio of $T \neq 0$ and $T=0$ correlators for the $\mathrm{S}$ - and P-wave charmonium at eight different values of $\beta$. As expected, temperature effects in the charmonium system are larger than that for bottomonium. Compared to bottomonium, which shows changes up to $\sim 1 \%$ for the S-wave at the highest temperature and up to $\sim 5 \%$ for P-wave [8], charmonium shows an increase up to $\sim 5 \%$ for the S-wave at the highest temperature and up to $\sim 13 \%$ for the $\mathrm{P}$-wave. Interestingly, both $\mathrm{S}$ - and $\mathrm{P}$-wave charmonium correlator ratios show non-monotonic behavior as the temperature increases. Both below and above $T_{c}\left(T_{c}=159 \mathrm{MeV}\right)$, the ratios for S- and P-wave charmonium are larger than one. As the temperature approaches $T_{c}$ from below, the ratios decrease toward unity. As the temperature further increases above $T_{c}$, the ratios also start to increase and to move further away from the value one. Such a non-trivial behavior may be understood in terms 

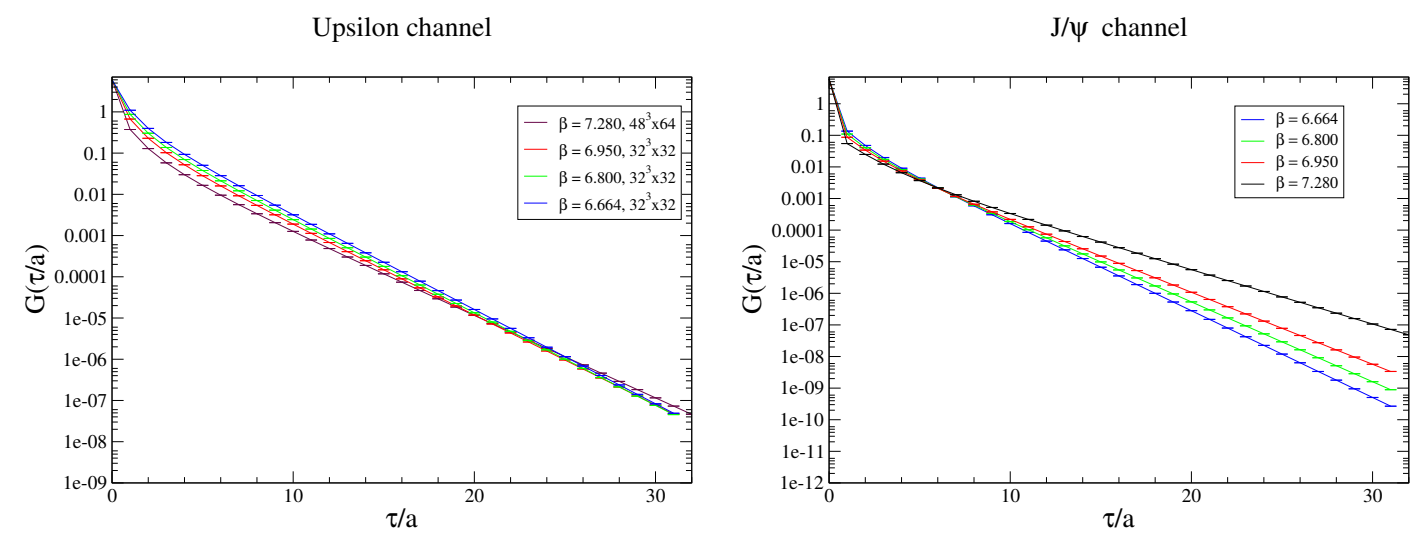

Figure 1: S-wave bottomonium correlator (left) [8] and S-wave charmonium correlator (right) at $T=0$
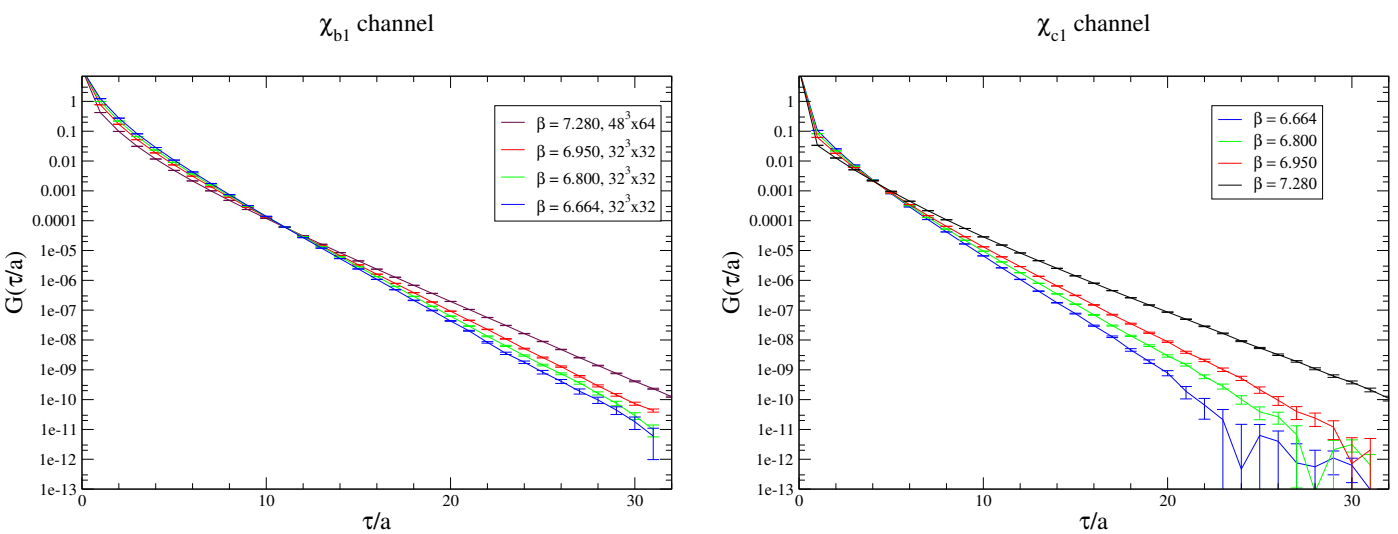

Figure 2: P-wave bottomonium correlator (left) [8] and P-wave charmonium correlator (right) at $T=0$

of an intricate interplay of the positions and widths of peaks in the underlying spectral functions. Lattice QCD based potential studies [17] may help to further shed light on these phenomena.

Let us turn to another measure of in-medium modification, the so called effective power $\gamma(\tau)$ defined from the normalized Euclidean time derivative of the NRQCD correlators

$$
\gamma(\tau)=\frac{\tau}{G(\tau)} \frac{d G(\tau)}{d \tau}
$$

Its values for the S-wave and P-wave channels at $T>0$ are shown in Fig. 4 and Fig. 5 respectively.

Each figure includes four sets of $\gamma(\tau)$. The dotted line denotes the value for the asymptotic behavior of the non-interacting NRQCD correlators, i.e. $3 / 2$ for the S-wave and $5 / 2$ for the P-wave [2]. Two curves are calculated from the correlators in the presence of finite $\mathrm{T}$ gauge fields at the lowest $\left(0.91 T_{c}\right.$, green square) and highest temperature (1.61T $T_{c}$, magenta square). Those curves belonging to non-interacting gauge fields on trivial lattices correspond to $T=0.91 T_{c}$ (i.e., calculated with $U_{\mu}(x)=1$ but $M_{c} a=0.7566$, blue circle) and $T=1.61 T_{c}$ (i.e., with $M_{c} a=0.4274$, red circle). The $\gamma(\tau)$ for free $\mathrm{S}$ - and P-wave bottomonium approaches the asymptotic value of the free theory for relatively small values of $\tau$, while $\gamma(\tau)$ for the interacting S- and P-wave bottominium are quite different from the free behavior. 

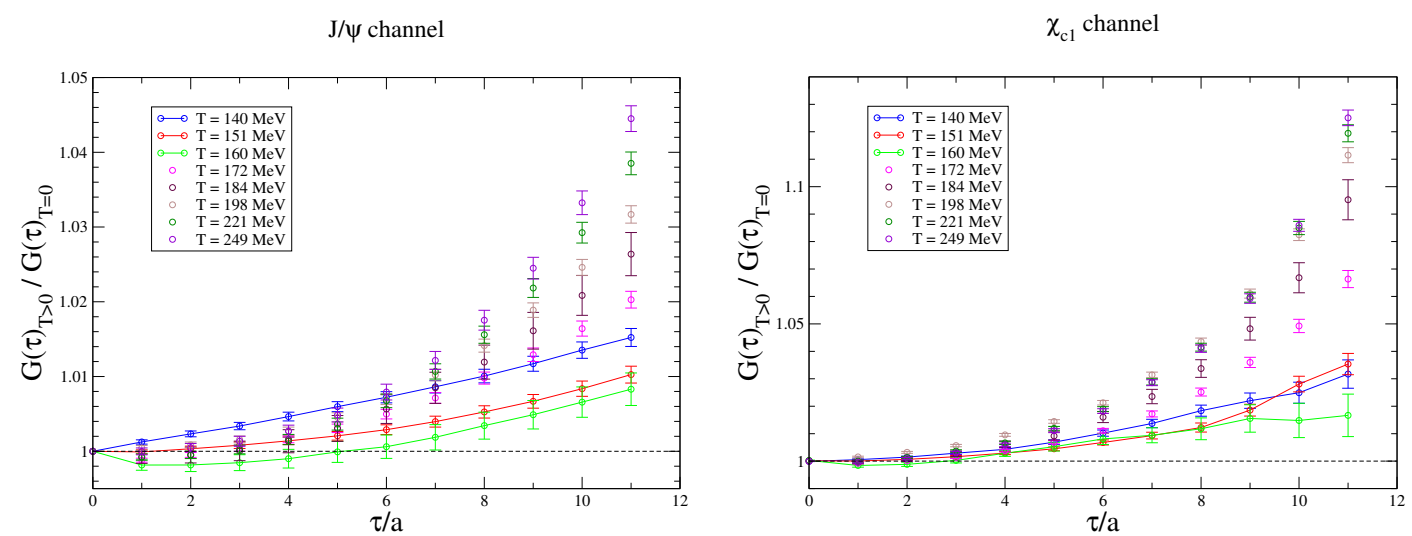

Figure 3: the ratio of S-wave charmonium correlator at $T \neq 0$ to $T=0 \mathrm{~S}$-wave charmonium correlator (left) and the ratio of $\mathrm{P}$-wave charmonium correlator at $T \neq 0$ to $T=0 \mathrm{P}$-wave charmonium correlator (right). the lines connecting the data points for $T \leq 160 \mathrm{MeV}$ are there just to guide.
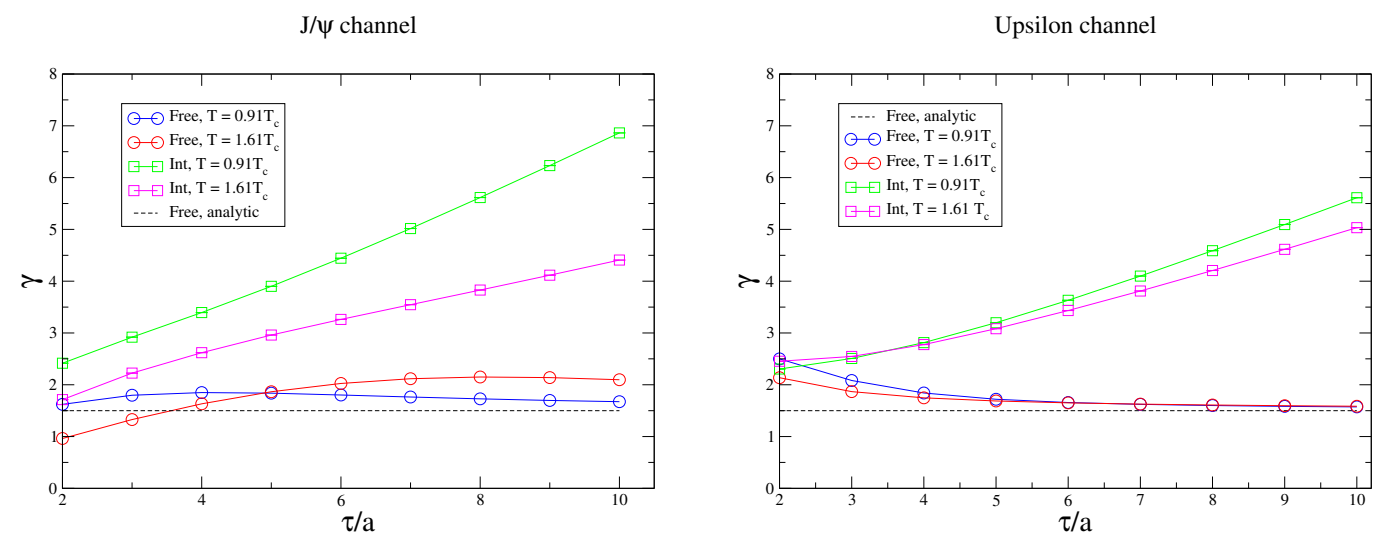

Figure 4: the effective power of S-wave charmonium correlator (left) and that of S-wave bottomonium correlator (right)

In the case of charmonium (both for S- and P-wave) the effective power $\gamma(\tau)$ corresponding to the free lattice correlator is different from the analytic result even for large $\tau$. These differences are larger at high temperatures. At $T=0.91 T_{c}$ the effective power is not very different for charmonium and bottomonium. At higher temperatures, i.e at $T=1.6 T_{c}$ there are significant differences. We see a larger temperature dependence of $\gamma$ for charmonium, and the value of $\gamma$ gets closer to the free theory result. This implies stronger modification of bound state properties and/or dissolution of the bound states in the charmonium sector. On the other hand $\gamma$ in the interection case is always quite different from the free case. This is most likely due to the non-trivial behavior of the spectral functions near the threshold [18]. Even if all bound states are melted the free spectral function is a poor approximation of the interacting quarkonium spectral function [18]. Furthermore, the interpretation of $\gamma(\tau)$ is quite subtle due to renormalization effects at the threshold near $2 M$ and dicretization effects in the quarkonium correlators [6]. The latter aspect is a less of a concern for our charmonium study, since the HotQCD gauge configurations are quite close to the continuum limit. 
$\chi_{\mathrm{c} 1}$ channel

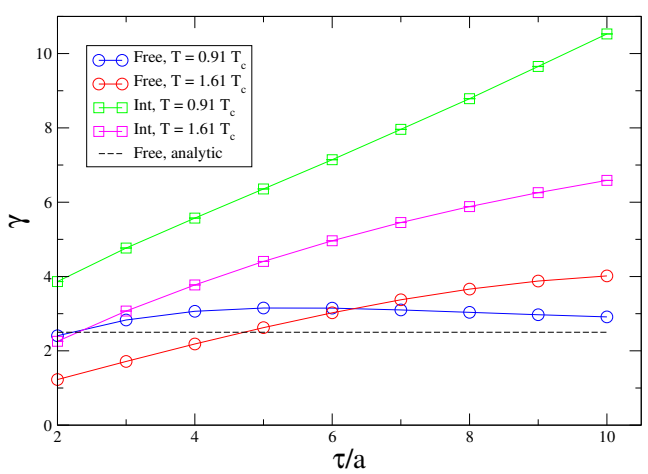

$\chi_{\mathrm{b} 1}$ channel

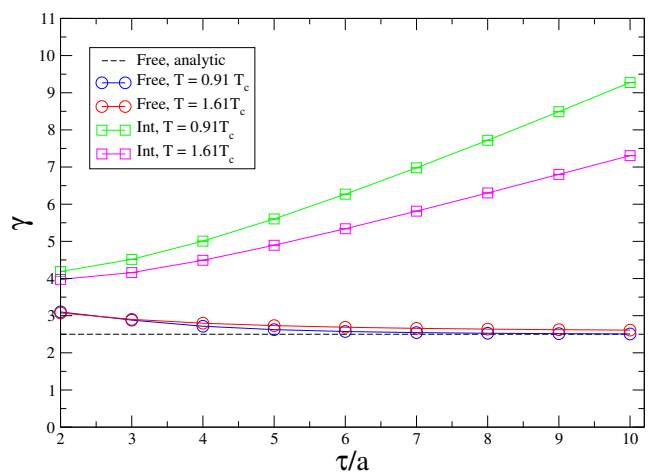

Figure 5: the effective power of P-wave charmonium correlator (left) and that of P-wave bottomonium correlator (right)

\section{Discussion}

S- and P-wave charmonium correlators are calculated using a lattice discretized formulation of NRQCD. In the ratio of non-zero temperature to $T=0$ correlators, a larger temperature effect is seen for charmonium system than bottomonium system. The size of temperature effect in $J / \psi$ is similar to that in $\chi_{b 1} \cdot \chi_{c 1}$ shows even larger temperature effect in the ratio.

The effective power $(\gamma)$ defined by the normailized Euclidean time derivative of the correlator suggests that the $T \neq 0 \mathrm{~J} / \psi$ channel is binding up to $T=1.61 T_{c}(=249) \mathrm{MeV}$, since $\gamma$ of $J / \psi$, calculated on the corresponding thermal gauge configuration behaves far differently from that of $J / \psi$ on a non-interacting gauge configuration. $\chi_{c 1}$ appears to be melting at $T=1.61 T_{c}(=249)$ $\mathrm{MeV}$, since $\gamma$ of $\chi_{c 1}$ calculated on the thermal gauge configuration behaves similar to that of $\chi_{c 1}$ on a trivial gauge configuration. Of course, since a charm quark is lighter than a bottom quark, the charmonium system may suffer from larger relativistic corrections. $M_{c} a<1$ (see Tab. 1) may cause sizeable radiative corrections in the coefficients of the NRQCD Lagrangian. Further study is required to understand the behavior of the effective power, in particular since renormalization effects around the threshold can obscure its meaning [6].

The reconstruction of spectral functions for S- and P-wave charmonium using both MEM and the new Bayesian method, as well as the investigation of their systematic uncertainties using increased statistics is under way.

\section{Acknowledgements}

SK is supported by the National Research Foundation of Korea grant funded by the Korean government (MEST) No. 2012R1A1A2A04668255. PP is supported by U.S.Department of Energy under Contract DE-SC0012704. NRQCD correlators on HotQCD configurations are computed using the LQCD Computing Facilities at Jefferson Lab. We thank the HotQCD collaboration for providing the gauge configurations for this study.

\section{References}

[1] T. Matsui and H. Satz, “J/ $\psi$ Suppression by Quark-Gluon Plasma Formation,” Phys. Lett. B 178 (1986) 416. 
[2] G. Aarts, S. Kim, M. P. Lombardo, M. B. Oktay, S. M. Ryan, D. K. Sinclair and J. -I. Skullerud, "Bottomonium above deconfinement in lattice nonrelativistic QCD," Phys. Rev. Lett. 106 (2011) 061602 [arXiv:1010.3725 [hep-lat]].

[3] G. Aarts, C. Allton, S. Kim, M. P. Lombardo, M. B. Oktay, S. M. Ryan, D. K. Sinclair and J. I. Skullerud, "What happens to the Upsilon and eta $b_{b}$ in the quark-gluon plasma? Bottomonium spectral functions from lattice QCD,” JHEP 1111 (2011) 103 [arXiv:1109.4496 [hep-lat]].

[4] G. Aarts, C. Allton, S. Kim, M. P. Lombardo, M. B. Oktay, S. M. Ryan, D. K. Sinclair and J. -I. Skullerud, "S wave bottomonium states moving in a quark-gluon plasma from lattice NRQCD," JHEP 1303 (2013) 084 [arXiv:1210.2903 [hep-lat]].

[5] G. Aarts, C. Allton, S. Kim, M. P. Lombardo, S. M. Ryan and J.-I. Skullerud, "Melting of P wave bottomonium states in the quark-gluon plasma from lattice NRQCD,” JHEP 1312 (2013) 064 [arXiv:1310.5467 [hep-lat]].

[6] G. Aarts, C. Allton, T. Harris, S. Kim, M. P. Lombardo, S. M. Ryan and J. I. Skullerud, "The bottomonium spectrum at finite temperature from $\mathrm{N}_{f}=2+1$ lattice QCD,” JHEP 1407 (2014) 097 [arXiv:1402.6210 [hep-lat]].

[7] S. Kim, P. Petreczky and A. Rothkopf, PoS LATTICE 2014 (2014) 208 [arXiv:1410.2110 [hep-lat]].

[8] S. Kim, P. Petreczky and A. Rothkopf, "Lattice NRQCD study of S- and P-wave bottomonium states in a thermal medium with $N_{f}=2+1$ light flavors," Phys. Rev. D 91 (2015) 054511 [arXiv:1409.3630 [hep-lat]].

[9] G. T. Bodwin, E. Braaten and G. P. Lepage, "Rigorous QCD analysis of inclusive annihilation and production of heavy quarkonium,” Phys. Rev. D 51 (1995) 1125 [Erratum-ibid. D 55 (1997) 5853] [arXiv:hep-ph/9407339].

[10] T. Umeda, “A constant contribution in meson correlators at finite temperature," Phys. Rev. D 75 (2007) 094502 [arXiv:hep-lat/0701005].

[11] G. Aarts and J. M. Martínez Resco, “Transport coefficients, spectral functions and the lattice,” JHEP 0204 (2002) 053 [arXiv:hep-ph/0203177].

[12] P. Petreczky, “On temperature dependence of quarkonium correlators,” Eur. Phys. J. C 62 (2009) 85 [arXiv:0810.0258 [hep-lat]].

[13] M. Asakawa, T. Hatsuda and Y. Nakahara, "Maximum entropy analysis of the spectral functions in lattice QCD,” Prog. Part. Nucl. Phys. 46 (2001) 459 [hep-lat/0011040].

[14] Y. Burnier and A. Rothkopf, "Bayesian Approach to Spectral Function Reconstruction for Euclidean Quantum Field Theories,” Phys. Rev. Lett. 111 (2013) 182003 [arXiv:1307.6106 [hep-lat]].

[15] A. Bazavov et al., "The chiral and deconfinement aspects of the QCD transition," Phys. Rev. D 85 (2012) 054503 [arXiv:1111.1710 [hep-lat]].

[16] A. Bazavov et al. [HotQCD Collaboration], "Equation of state in ( 2+1 )-flavor QCD," Phys. Rev. D 90, no. 9, 094503 (2014) [arXiv:1407.6387 [hep-lat]].

[17] Y. Burnier, O. Kaczmarek and A. Rothkopf, "Quarkonium at finite temperature: Towards realistic phenomenology from first principles,” arXiv:1509.07366 [hep-ph].

[18] A. Mocsy and P. Petreczky, "Can quarkonia survive deconfinement?," Phys. Rev. D 77, 014501 (2008) [arXiv:0705.2559 [hep-ph]]. 